\title{
Error analysis in gravity aided navigation
}

\author{
Wei Hongwei ${ }^{1}$, Wu Meiping ${ }^{1}$, Tie Junbo ${ }^{1}$ and Wang Shenquan ${ }^{2}$ \\ ${ }^{1}$ College of Mechatronics Engineering and Automation, National University of Defense Technology, Changsha 410073, China, \\ ${ }^{2}$ Manned Space System Research Center, Beijing100094, China,
}

\begin{abstract}
For underwater vehicles, because GPS signals are not always available, it is necessary to use inertial navigation system (INS) for navigation. In order to improve the navigation performance, the gravity value calculated by the gravity field model is introduced into the navigation system to reduce the influence of gravity on the navigation accuracy. However, due to the difference between the actual gravity field and the gravity field model, the navigation error caused by gravity field still exists. In this paper, this error is studied based on the theoretical analysis and simulation. The results show that the accuracy of gravity-aided navigation will decrease sharply when the position error of the underwater vehicles reach $9.6 \mathrm{n}$ miles, because at this moment, the calculation error of vertical deflection is the largest. In the simulation experiment, the position error of the gravity-aided navigation system reach $9.6 \mathrm{n}$ miles in 44 hours. Therefore, for the underwater vehicles navigation system, it is recommended that the vehicles should be corrected once every 44 hours.
\end{abstract}

\section{Introduction}

As a basic physical field, gravity field restricts all physical events of the earth itself and its adjacent space [1]. The inertial navigation system is also affected by the earth gravity field, and the navigation accuracy of inertial navigation system is closely related to the value of gravity.

As the accuracy of inertial transducers increase, the influence of gravity becomes the main factor limiting the precision of high accuracy inertial navigation system [2]. Therefore, in inertial navigation system, the influence of gravity field need to be eliminated through reasonable compensation method. In the low-precision inertial navigation system, Equation (1) is often used to calculate the value of gravity, and the influence of gravity field on inertial navigation system is compensated using this value [3].

$$
\gamma=\frac{\gamma_{0}}{\left(1+h / R_{0}\right)^{2}}
$$

in which:

$$
\begin{aligned}
& \gamma_{0}=9.780318\left(1+5.3024 \times 10^{-3} \sin ^{2} L-5.9 \times 10^{-6} \sin ^{2} 2 L\right) \\
& R_{0}=\sqrt{R_{M} R_{N}}
\end{aligned}
$$

The radius of curvature in meridian $R_{M}$ and the radius of curvature in prime vertical $R_{N}$ can be calculated by the following equations:

$$
\left\{\begin{array}{l}
R_{M}=R\left(1-e^{2}\right) /\left(1-e^{2} \sin ^{2} L\right)^{3 / 2} \\
R_{N}=R /\left(1-e^{2} \sin ^{2} L\right)^{1 / 2}
\end{array}\right.
$$

where $R$ is semi-major axis of the reference spheroid; $e$ represents the major eccentricity of the reference spheroid; $L$ is latitude of the inertial navigation system and $h$ is the height above ground.

In the above-mentioned compensation method, it is considered that the direction of the gravity vector coincides with the direction of the reference ellipsoid normal. In fact, the reference ellipsoid norm is not consistent with the direction of the geoid normal, so this compensation method cannot completely eliminate the influence of the gravity field on the inertial navigation system.

The angle between the reference ellipsoid norm and the geoid normal is called the vertical deflection. The results of JIN Ji-hang's research indicate that the position error of the inertial navigation system caused by vertical deflection is about $3 \mathrm{~km}$ on the set route [4].

In order to improve the accuracy of the gravity compensation method, Zhao Zhong et al. analyzed the effect of vertical deflection on high accuracy inertial navigation system [5]. Tie Jun-bo et al. improved the 
method of calculating gravity. He introduced EGM2008 into the inertial navigation system[6]. EGM2008 is Earth's gravitational model with very high resolution, which has been released by the Naional GeospatialIntelligence Ageny (NGA) Development Team [7]. EGM2008 is complete to spherical harmonic degree and order 2159, and it contains additional coefficients extending to degree 2190 and order 2159.

Based on EGM2008, the horizontal gravity disturbance can be calculated. Introduce the horizontal gravity disturbance into the inertial navigation system, and then the accuracy of inertial navigation positioning can be increased by $120 \mathrm{~m} / 4 \mathrm{~h}$ [6].

The compensation method that using vertical deflection, which is achieved from the horizontal gravity disturbance that calculated using EGM2008, to assist inertial navigation can effectively reduce the error of the navigation system and improve the navigation accuracy. Nevertheless, this compensation method does not eliminate the errors caused by gravity field. In this paper, we will study the error in this compensation method, and analyse the distribution of vertical deflection based on EGM2008. Finally, based on the simulation experiment, the optimal time for the underwater vehicle to be corrected is given.

\section{Calculation of vertical deflection}

In order to study the distribution of vertical deflection, the value of vertical deflection should be calculated based on EGM2008. The calculation steps are shown in figure 1: First, calculate the horizontal gravity disturbance with EGM2008, and then the vertical deflection is calculated by the relationship between horizontal gravity disturbance and vertical deflection.

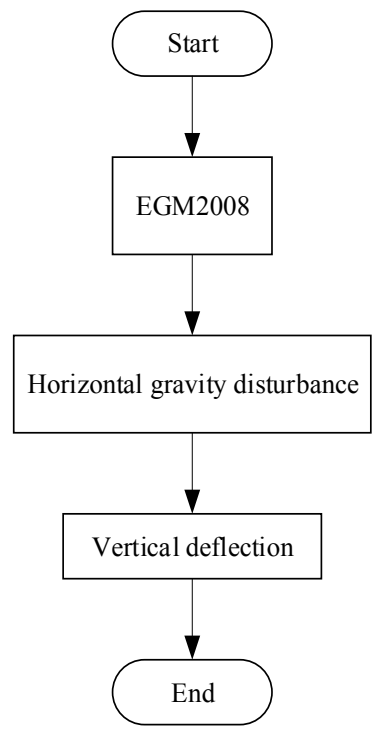

Figure 1. Calculation steps of vertical deflection.

\subsection{Relationship between horizontal gravity disturbance and vertical deflection}

The prime vertical component of vertical deflection is denoted by $\eta$ (rad), and the meridional component of vertical deflection is denoted as $\varepsilon$ (rad). The meridional component and prime vertical component of gravity disturbances are denoted by $\delta g_{N}$ and $\delta g_{E}$, respectively.

There is a relationship between them as follows[6]:

$$
\begin{aligned}
& \delta g_{N}=-\gamma \cdot \eta \\
& \delta g_{E}=-\gamma \cdot \varepsilon
\end{aligned}
$$

where $\gamma$ is the normal gravity, which can be calculated by equation (1).

\subsection{Horizontal gravity disturbance}

Horizontal gravity disturbances can be calculated, using EGM2008 as follows:

The perturbed potential of gravity is denoted by $T$, since it is a harmonic function. It can be expressed by the following equation:

$T=\frac{G M}{r} \sum_{n=2}^{N}\left(\frac{a}{r}\right)^{n} \sum_{m=0}^{n}\left(\bar{C}_{n m}^{*} \cos m \lambda+\bar{S}_{n m} \sin m \lambda\right) \bar{P}_{n m}(\cos \theta)$

in which $G M$ and $a$ are fundamental parameters of WGS-84, the values of them are shown in Table $1 . \bar{C}_{n m}^{*}$ and $\bar{S}_{n m}$ are spherical harmonic coefficients corresponding to the fully normalized Legendre functions, which are provided by EGM2008.

Table 1. Fundamental parameter of WGS-84.

\begin{tabular}{|c|c|}
\hline Parameter & Value \\
\hline$a$ & 6378136.3 \\
\hline$f$ & $1 / 298.256415099$ \\
\hline$G M$ & $0.3986004415 \times 10^{20}$ \\
\hline$\omega_{i e}$ & $7292115 \times 10^{-11}$ \\
\hline
\end{tabular}

The relationship between the perturbed potential of gravity and the horizontal gravity disturbance is as follows [8-9]:

$$
\begin{aligned}
& \delta g_{N}=-\frac{1}{\rho} \frac{\partial T}{\partial \theta} \\
& \delta g_{E}=-\frac{1}{\rho \cdot \sin \theta} \frac{\partial T}{\partial \lambda}
\end{aligned}
$$

in which $\delta g_{N}$ and $\delta g_{E}$ are northward and eastward horizontal gravity disturbance, respectively.

Substituting equation (5) into equation (6) yields:

$$
\begin{aligned}
& \delta g_{N}=-\frac{G M}{r^{2}} \sum_{n=2}^{\infty}\left(\frac{a}{r}\right)^{n}\left(\bar{C}_{n m}^{*} \cos m \lambda+\bar{S}_{n m} \sin m \lambda\right) \frac{d \bar{P}_{n m}(\cos \theta)}{d \theta} \\
& \delta g_{E}=-\frac{G M}{r^{2} \sin \theta} \sum_{n=2}^{\infty}\left(\frac{a}{r}\right)^{n} \sum_{m=0}^{n} m\left(-\bar{C}_{n m}^{*} \sin m \lambda+\bar{S}_{n m} \cos m \lambda\right) \bar{P}_{n m}(\cos \theta)
\end{aligned}
$$


Then, the horizontal gravity disturbance can be calculated by equation(7).

\section{Analysis of vertical deflection}

According to the above methods, the vertical deflection in the range of $10^{\circ} \mathrm{N}-11^{\circ} \mathrm{N}, 110^{\circ} \mathrm{E}-112^{\circ} \mathrm{E}$ is shown in figure 2-3.

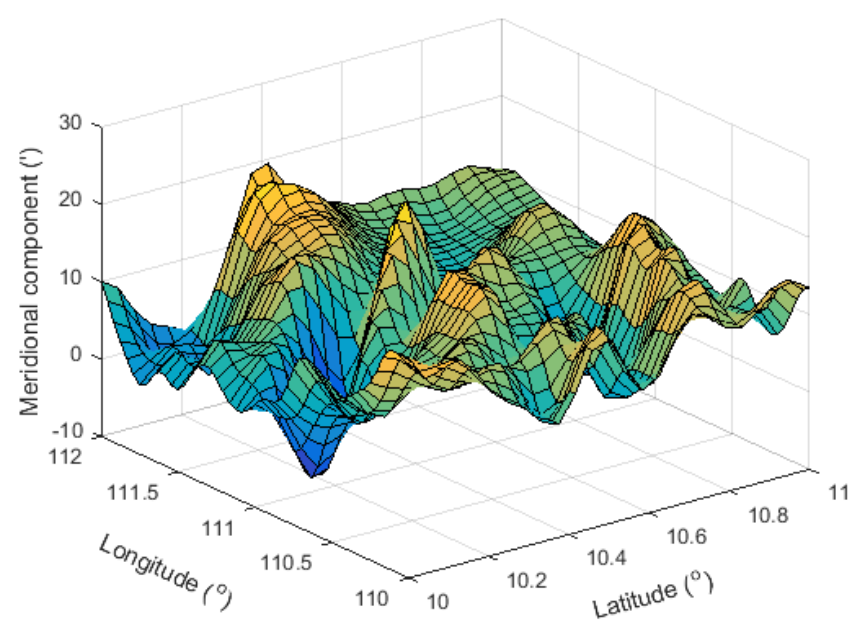

Figure 2. Meridional component of the vertical deflections in arc-seconds

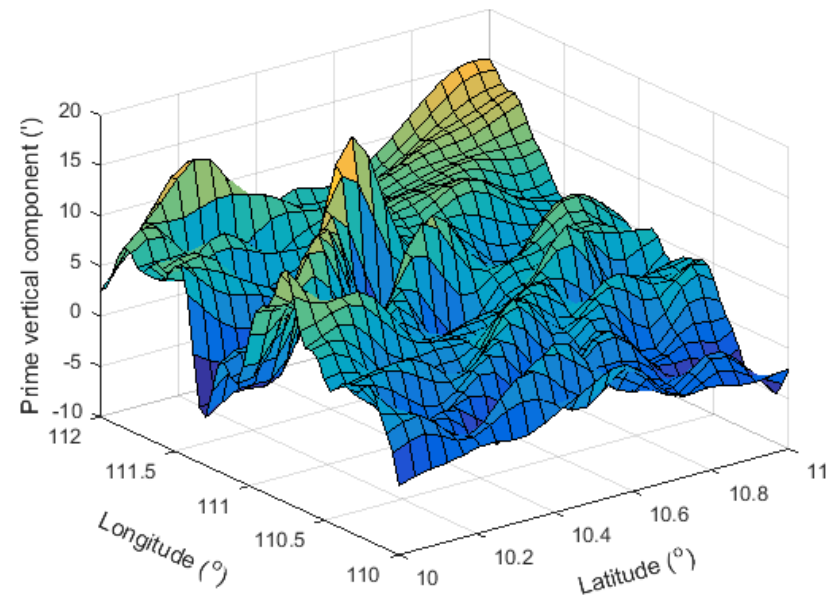

Figure 3. Prime vertical component of the vertical deflections in arc-seconds

Then the vertical deflections in a survey line is analyzed. The range of the survey line is $111^{\circ} \mathrm{E}$, $10^{\circ} \mathrm{N}-11^{\circ} \mathrm{N}$. The vertical deflection of this survey line is shown in figure 4.

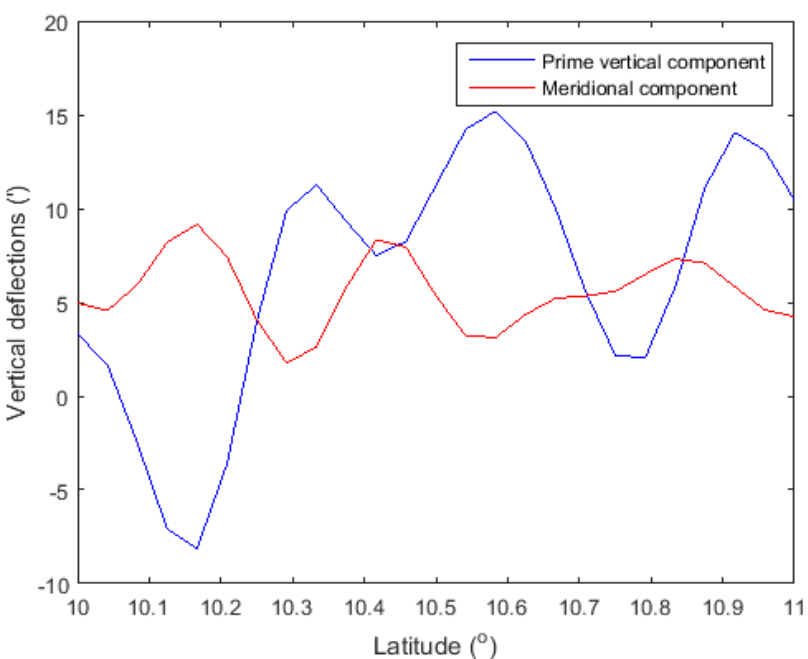

Figure 4. The vertical deflections in the survey line

As shown in figure 4, the blue line represents the prime vertical component in the survey line, and the red line represents the meridional component in the survey line. It can be seen that the periodicity of the two components of the vertical deflection is about 0.32 degrees.

For the survey line of $10.5^{\circ} \mathrm{N}$ and $110^{\circ} \mathrm{E}-112^{\circ} \mathrm{E}$, the conclusion above also holds.

Therefore, it can be concluded that for gravity aided inertial navigation, if the position error reaches $9.6 \mathrm{n}$ miles, then the error of the calculated vertical deflection is maximized. That is to say, if continue using gravity aided navigation, it will lead to greater error. In this case, the gravity aided navigation system needs to be corrected.

\section{Simulation}

Based on the simulation experiment, the precision of gravity aided navigation is studied in this section. Because of the lack of inertial navigation data of underwater vehicles, a high-precision strapdown inertial navigation system for ship is used to replace the navigation system for underwater vehicle.

In the following simulation experiment, the data of a shipboard laser strapdown inertial navigation system is used as the raw data for simulation; the position calculated by GPS precision single point positioning is used as an accurate value for reference.

Simulation lasted 48 hours, first, the raw data was used to calculate the position of the carrier in the inertial navigation system. In the calculation, it is assumed that the projection of gravity vector in the $\mathrm{X}$ axis and $\mathrm{Y}$ axis direction of navigation coordinate system is zero. And the projection of gravity vector in $\mathrm{Z}$ axis direction is calculated by equation (1) and equation (2). The position error of the inertial navigation system is obtained by comparing the position calculated by the GPS with the position information of the inertial navigation system.

Next, based on the model of earth gravity field EGM2008, the value of vertical deflection was calculated. And then the value of vertical deflection was used to compensate for the influence of horizontal components in the gravity field on the inertial navigation system. By this 
compensation, the gravity field used in the inertial navigation system will be closer to the real gravity field, and the error will be smaller. Such inertial navigation system is known as gravity assisted inertial navigation system. In the same way, the position obtained by the gravity-assisted inertial navigation system is compared with the position calculated by the GPS to obtain the position error of the gravity-assisted inertial navigation system.

The navigation position errors that using gravity aided navigation and without gravity aided navigation are shown in figure 5. The blue line indicates the position error of the navigation system using gravity aided, and the red line indicates the position error of the navigation system without gravity aided. It can be seen that the position error that using gravity aided navigation is less than the error that without gravity aided navigation.

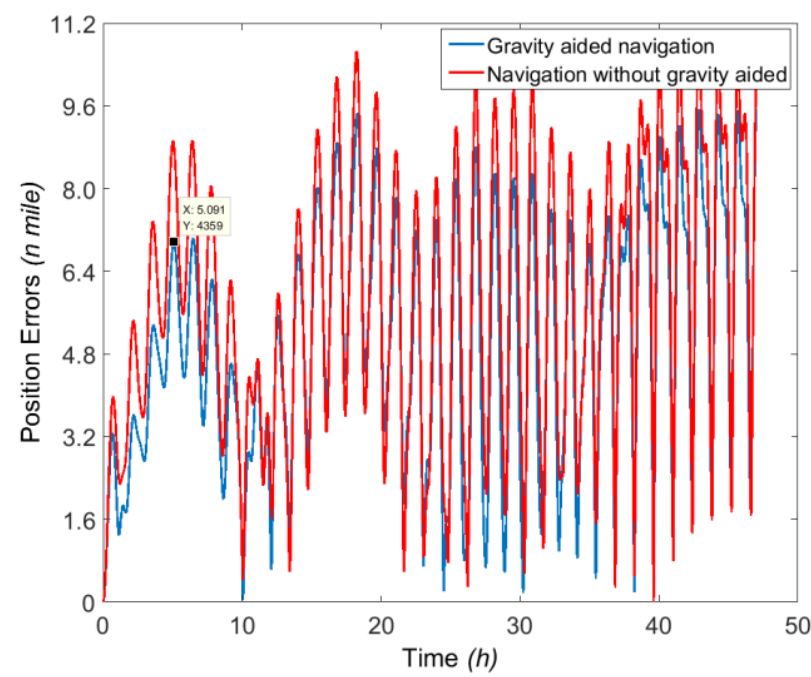

Figure 5. Position errors of navigation system

After 44 hours, the position error of the gravity aided navigation system reached $9.6 \mathrm{n}$ miles. At this point, the error of the vertical deflection reaches the maximum, and it will produce a larger navigation error if continue using the gravity aided navigation system for navigation. Therefore, the navigation system needs to be corrected at this time to keep the navigation accuracy.

\section{Conclusion}

In this paper, the errors in gravity assisted inertial navigation system for underwater vehicle is analysed. Furthermore, the errors caused by using the gravity field model EGM2008 to calculate the vertical deviation and its influence on inertial navigation system are studied.

Through the above analysis and further simulation experiments, we can get the conclusion that when the position error reaches $9.6 \mathrm{n}$ miles, the error in calculation of gravity reaches the largest. Therefore, the gravity assisted navigation system should be corrected when the position error reaches $9.6 \mathrm{n}$ miles.

According to the results of this paper, we can calculate the optimal time to calibrate the gravity assisted inertial navigation system based on the actual performance of the underwater vehicle. In this way, we can make the underwater vehicle voyage as long as possible under the condition that the navigation accuracy is guaranteed.

With the conclusions of this paper, the navigation accuracy of underwater vehicle can be improved and the rapid growth of the navigation error can be effectively suppressed.

\section{Acknowledgements}

We would like to express our gratitude to all those who helped us during the writing of this paper. And this paper is supported by the National Key R\&D Program of China (2017YFC0601701).

\section{References}

1. K.D. Zhang, Research on the Methods of Airborne Gravimetry Based on SINS/DGPS. Changsha, Hunan, P.R.China, National University of Defense Technology, (2007)

2. CSIT. Overview of present state and trends for inertial technology. Report on Advances in Inertial Technology, Beijing, 12-15 (2010)

3. D.H. Titterton, Strapdown Inertial Navigation Technology(2nd edition): American Institute of Aeronautics and Astronautics,Inc. (2004)

4. J.H Jin, S.F. Bian, Analysis of inertial navigation system positioning error caused by gravity disturbance. Geomatics and Information Science of Wuhan University, 35(1): 30-32 (2010)

5. Z. Zhao, P. Wang, Analysis and compensation of vertical deflection effect on high accuracy inertial navigation system. Journal of Chinese Inertial Technology, 23(6): 701-705 (2013)

6. J.B. Tie, M.P. Wu, S.K. Cai, K.D. Zhang, J.X. Lian, Gravity disturbance compensation method based on Earth Gravitational Model 2008. Journal of Chinese Inertial Technology. (to be published)

7. Nikolaos K. Pavlis, Simon A. Holmes, Steve C. Kenyon, John K. Factor; The development and evaluation of the Earth Gravitational Model 2008 (EGM2008), Journal of Geophysical Research: Solid Earth (1978-2012) Volume 117, Issue B4, April (2012)

8. Rapp, R. H. A FORTRAN program for the computation of gravimetric quantities from high degree spherical harmonic expansions. Report No. 334. Ohio State University, Dept. of Geodetic Science and Surveying, Columbus, Ohio (1982).

9. Colombo, O. L. Numerical methods for harmonic analysis on the sphere. Report No. 310. Dept. of Geodetic Science, The Ohio State University, Columbus, Ohio

(1981) 